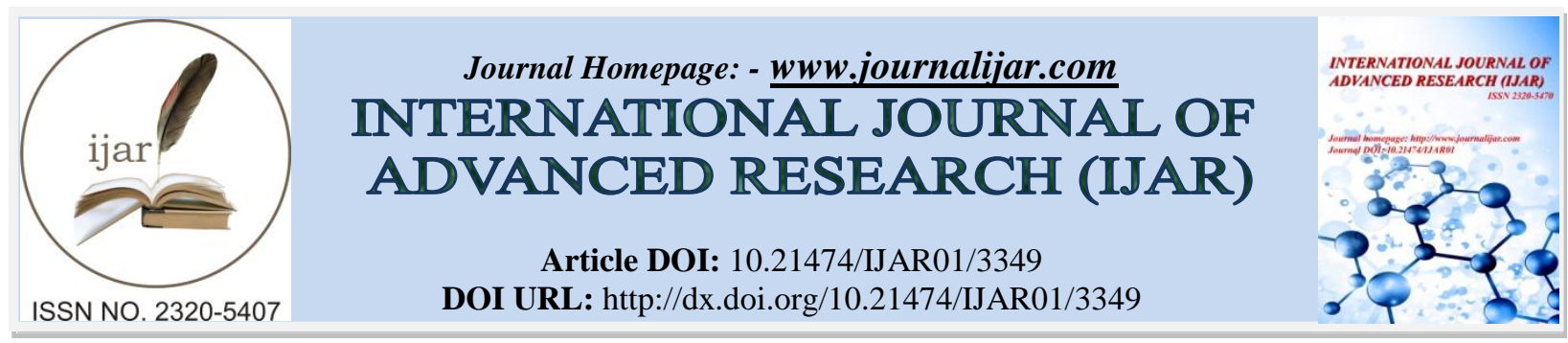

RESEARCH ARTICLE

\title{
ANALYSIS OF HYPOLIPIDEMIC EFFECT OF RHINACANTHUS NASUTUS(LINN) KURZ IN HIGH FAT DIET INDUCED HYPERLIPIDEMIC RAT.
}

\author{
D. Sajeda, K. Jayalakshmi and K. Thyagaraju. \\ Professor, Reasearch Supervisor, Sri Venkateswara University, Tirupathi-517503, AP, India.
}

\section{Manuscript Info}

Manuscript History

Received: 25 December 2016

Final Accepted: 05 January 2017

Published: February 2017

Key words:-

Hyperlipidemia, Medicinal plant

Rhinacanthus nasutus , High fat diet.
Abstract

Objective: To evaluate the hypolipidemic effect of Rhinacanthus nasutus (R.nasutus) in high fat diet induced (HFD) hyperlipidemic rats. Methods: The methanol leaf extract of R.nasutus was tested for hyperlipidemic effect in the albino rats at the selected optimum dosage of $200 \mathrm{mg} / \mathrm{kg}$ body weight orally. Adult male albino rats of six numbers in each group were undertaken to study, and evaluated. Results: In group II animals, fed with high fat diet the activity levels of serum total cholesterol (TC), triglycerides (TG), low density lipoprotein (LDL), very low density lipoprotein-cholesterol (VLDL) were significantly enhanced when compared to normal rat. Rhinacanthus nasutus methonolic leaf extract (RNM) group III treated rats showed depleted levels of the lipid molecules.

Conclusion: It could be concluded that the methanol leaf extract of R.nasutus exhibited a significant hypolipidemic effect on HFD induced hyperlipidemia in rats.

Copy Right, IJAR, 2017,. All rights reserved.

\section{Introduction:-}

Diseases of the cardiovascular system are the most common cause of death. Lifestyle changes have a significant impact on the health of the people. The modernization of societies appears to result in a dietary pattern that is high in saturated fats, refined sugars and is low in fiber content. (Brai, et al. 2007) [1]. It is now established that hyperlipidemia represents a major risk factor for the premature development of atherosclerosis and its cardiovascular complications. Hyperlipidemia is a disorder characterized by an increase in blood lipoprotein or cholesterol levels. Atherosclerosis is a common condition in both developed and developing countries and is recognized to be an inflammatory condition leading to the development of ischemic heart diseases, cerebrovascular diseases and peripheral vascular diseases. Cardiovascular diseases (CVD) account for $31 \%$ deaths worldwide in 2015(WHO 2015) [2]. The major risk factor of CVD is hypertension, hypercholesterolemia, diabetics and obesity. The death of UK people due to heart problems were 28 and 26, in male and females, respectively, in 2015. Hypertensive individual number in India is projected to increase two fold by 2025. (Kearney, et al. 2005) [3].

Hyperlipidemia contributes drastic threat towards the spread and expansion of atherosclerosis and coronary heart diseases (CHD). Significant increase of lipid profiles is responsible for the onset of CHD. Ischemic heart disease is a major risk factor in the pathogenesis of preoperative adverse cardiovascular events which lead to significant morbidity and mortality within the high risk surgical patient population.(Howard-Alpe,et al, 2006) [4]. Recent studies have shown that lipid associated disorders are not only attributed to the total serum cholesterol, but also to its distribution among different lipoproteins. The low density lipoproteins (LDL) are the major carriers of cholesterol 
towards tissues having atherogenic potential, while the high density lipoproteins (HDL) carry cholesterol from peripheral tissues to the liver. HDL thus gives protection against many cardiac problems and obesity, although genetic factors recline behind these lipid disorders.(Sattivel.et al,2000) [5]. The treatment of hyperlipidemia depends on the patient's cholesterol profile. Many antihyperlipidemic agents like statins, fib rates, niacin, bile acids, ezitimibe etc. reduce cholesterol level in different conditions. (Durrington et al 2003)[6].

Currently available hypolipidemic drugs have been associated with a number of side effects. The consumption of synthetic drugs leads to hyperuricemia, diarrhoea, nausea, myositis, gastric irritation, flushing, dry skin and abnormal liver function.(Speight.et al 1987) [7]. Only two drugs, orlistat and sibutramine, have been approved for long-term use in significantly obese patients by the U.S. Food and Drug Administration. The other drugs showed adverse effects including gastro-intestinal discomforts, flatulence, and diarrhea.

Since ancient times plants have been exemplary sources of medicine because they constitute raw materials as drugs for treating various ailments of human beings. So, there has been significant development in the fields of synthetic drug chemistry and antibiotics based on plant products isolation and characterization. During the last two decades, considerable changes have taken place in the medicinal system all over the world. Because of the general awareness of the widespread toxicity and harmful after-effects associated with the long term use of synthetic drugs and antibiotics, drugs from natural sources are being preferred.

According to the World Health Organization (WHO 1977) [8], a medicinal plant is a plant in which, one or more of its organs contains substances that can be used for the synthesis of useful drugs. This definition distinguishes those plants whose therapeutic properties and constituents have been established scientifically from plants that are regarded as medicinal but which have not yet been subjected to thorough investigation. The term herbal drug determines the part/parts of a plant used for preparing medicines (for example: leaves, flowers, seeds, roots, barks, stems, etc.).

Medicinal plants contain biologically active chemical substances such as saponins, tannins, essential oils, flavonoids, alkaloids and other secondary metabolites.( Harborne. et al, 1973, Harborne. et al,1996) [9,10], which have curative properties to almost all living organisms. These complex chemical substances has reported that plants also contain certain other compounds that moderate the effects of the active ingredients. The plant derived preparations have long been used as traditional remedies and in folklore medicine for the treatment of hyperlipidemia in many parts of the world.

Recently, the search for appropriate antihyperlipidemic agents have been again focused on plants because of less toxicity, easy availability and absorption in the body so that they may be better for treatment than currently used drugs. Traditional system of medicine like Ayurveda, Unani and Chinese prescribe numerous herbal drugs for CVD.

At present herbal hypolipidemics related to plants have gained importance to fill the lacunae created by herbal drugs because they are generally considered to be less toxic and less prone to side effects than drugs manufactured by chemical synthesis. The potential therapeutic and preventive benefits of plant-based medications have been the subject of extensive studies, and many natural constituents have been uncovered with significant pharmacologic activity, including agents with antiglycemic, hypolipidemic and antihypertensive properties.( Kumari, Son. et al, 2007) $[11,12]$.

Rhinacanthus nasutus (Linn) Kurz, a medicinal plant, belongs to Acanthaceae family, is a shrub and is well known for its medicinal uses, commonly called as Nagamalli in Telugu. This plant is widely distributed in some parts of the subcontinent India and in the region of Southeast Asia and China. The plant is small, slender, shrub and 1-2 meter height. Various parts of this plant have been used for the treatment of eczema, pulmonary tuberculosis, herpes, hepatitis, diabetes, hypertension, skin diseases, ring worms, cancer, scurvy, leprosy and obesity. The plant leaves, roots and seeds also used as an antidote for snake bites.(Visweswara.et al 2010, Yesilbursad et al, 2005) [13-15]. In the present study, we investigated the effect of R.nasutus methanol leaf extracts on the reduction of cholesterol level in serum of wistar rats. 


\section{Materials and Methods:-}

Plant material:-

The fresh whole plant of Rhinacanthus nasutus (Linn) Kurz were collected from Tirumala forest, Tirupathi, Chittoor (Dt) Andhra Pradesh, India, identified and authenticated by Department of Botany, SV University, Tirupati.

\section{Plant extracts preparation:-}

The Rhinacanthus leaves were collected, washed through tap water and shade dried at room temperature and made powder using grinder. The powdered plant material was macerated with hexane, ethyl acetate, methanol and water, separately. The extract of each was then filtered with filter paper (Whatman No. 1) and the filtrates was kept under reduced pressure using rota evaporator at $40^{\circ} \mathrm{C}$ was concentrated and stored for further study at $4^{\circ} \mathrm{C}$. Preliminary screening test of each extract powder was performed at dosages of 50, 100, 150, 200, mg/kg body weight of rat. Among the four extracts, methonolic extract found to contain much of hyperlipidemic activity. Hence, the methanol leaf extract was selected and tested for hypolipidemic effect in the wistar rats at the selected optimum dosage of $200 \mathrm{mg} / \mathrm{kg}$ body weight and administered orally.

\section{Animals:-}

Adult male albino rats of wistar strain weighing about $120 \pm 15 \mathrm{~g}$ were purchased from Sri Venkateswara Enterprises, Bangalore. The animals were kept in polypropylene cages (six in each cage) at an ambient temperature of $(25 \pm 2){ }^{\circ} \mathrm{C}$ and at 55\%-65\% relative humidity. A $(12 \pm 1) \mathrm{h}$ ) light and dark schedule was maintained in the animal house till the animals were acclimatized to the laboratory conditions. They were fed with commercially available rat chow (Hindustan Lever Ltd., Bangalore. India) and had free access to water. The experiments were designed and conducted in accordance with the ethical committee.

\section{Development of high fat diet fed rats:-}

Rat was fed with two dietary regiment such as normal pellet diet (NPD) and high fat pellet diet (HFD). The rats were fed either with NPD or HFD (58\% fat, 25\% protein and 17\% carbohydrate, as a percentage of total kcal) adlibitum respectively, for the initial period of 2 weeks. (Srinivasan. et al, 2004) [16]. The composition and preparation of HFD were made as per the method (Reed et al, 2000).[17].

\section{Experimental Design:-}

The studies were conducted in six groups as said below by taking six animals in each group.

Group 1: Control animals fed with normal diet.

Group 2: High fat prepared diet fed rats.

Group 3: Both HFD +RMLE 200mg/kg b.wt fed rats.

Group 4: Both HFD+Orlistat $25 \mathrm{mg} / \mathrm{kgb} . w t$ drug as a standard fed rats.

Group5: RNMLE alone as plant product control.

Group6: Orlistat alone as drug control.

All feeding studies were made for six weeks of period. The dose material was suspended in distilled water and given orally using a gastric gavage.

\section{Collection of blood and preparation of serum samples:-}

At the end of the experimental period, blood samples were collected from the retro orbital plexus in vials. The blood samples were allowed to clot for $10 \mathrm{~min}$ at room temperature and then centrifuged at $3500 \mathrm{xg}$ for $15 \mathrm{~min}$. The clear, non heamolysed serum samples thus obtained were collected and stored at $-20^{\circ} \mathrm{C}$ until biochemical assays were carried out.

\section{Estimation of biochemical constituents of lipids in rats serum:-}

The biochemical estimations were carried out in our lab by using the following methods. Serum total cholesterol CHOD-POD method [18], triglyceride GPO-POD method using ethyl-N-(3- sulfopropyl)-m-anisidine sodium (ESPAS) [19], serum high density lipoprotein, serum low density lipoprotein and serum very low density lipoprotein by CHO-Phospho method [20].

\section{Statistical Analysis:-}

The results were expressed in mean \pm standard deviation. Statistical analysis was carried out by using one way ANOVA as in standard statistical software package of social science (SPSS). 


\section{Results:-}

To study the hypolipidemic effect of the methanolic leaf extract of R.nasutus on the high fat diet induced male albino rats, the levels of serum total cholesterol (TC), triglycerides (TG), low density lipoprotein (LDL), serum high density lipoprotein (HDL) and very low density lipoprotein-cholesterol (VLDL-C) were analysed in experimental animals. In group II animals, the levels of serum total cholesterol, triglycerides, low density lipoprotein and very low density lipoprotein cholesterol were significantly elevated as compared to that of normal groups, when they were fed with HFD (Table 1). On the other hand the serum level of serum high density lipoproteins (HDL) were significantly depleted in the HFD fed rat.

In group- III R.nasutus (200 mg/kg body weight) supplemented later with HFD fed rat showed a significant depletion in the levels of total cholesterol, triglycerides, low density lipoprotein and very low density lipoproteincholesterol as compared to normal group (Table:1). The level of HDL was significantly augmented in plant extract treated group.

Table 1:- Effect of the methanolic leaf extract of R.nasutus on serum lipid profile levels (mg/dL), in HFD induced rat.

\begin{tabular}{|c|c|c|c|c|c|c|}
\hline Group & Treatment & $\begin{array}{c}\text { Total cholesterol } \\
\mathrm{mg} / \mathrm{dl}\end{array}$ & $\begin{array}{c}\text { Tri- } \\
\text { Glycerides } \\
(\mathrm{mg} / \mathrm{dl})\end{array}$ & $\begin{array}{c}\text { HDL-C } \\
(\mathrm{mg} / \mathrm{dl})\end{array}$ & $\begin{array}{c}\text { LDL-C } \\
(\mathrm{mg} / \mathrm{dl})\end{array}$ & $\begin{array}{c}\text { VLDL-C } \\
(\mathrm{mg} / \mathrm{dl})\end{array}$ \\
\hline I & Control & $88.2 \pm 4.29^{*}$ & $74.73 \pm 5.02^{*}$ & $42.32 \pm 4.62^{*}$ & $33.65 \pm 6.0^{*}$ & $13.81 \pm 1.65^{*}$ \\
\hline II & HFD & $195.9 \pm 10.90^{*}$ & $109.2 \pm 8.25^{*}$ & $34.97 \pm 4.50^{*}$ & $141.5 \pm 13.20^{*}$ & $21.59 \pm 1.04^{*}$ \\
\hline III & $\begin{array}{c}\text { RNM+HFD } \\
(200 \mathrm{mg} / \mathrm{kg})\end{array}$ & $154.28 \pm 10.81^{* *}$ & $89.32 \pm 4.91^{* *}$ & $43.27 \pm 4.69^{* *}$ & $75.28 \pm 11.14^{* *}$ & $17.32 \pm 0.95^{* *}$ \\
\hline IV & $\begin{array}{c}\text { Orlistat+ } \\
\text { HFD } \\
(25 \mathrm{mg} / \mathrm{kg})\end{array}$ & $107.1 \pm 10.62^{* *}$ & $80.26 \pm 4.07^{*}$ & $45.07 \pm 4.71^{* *}$ & $46.41 \pm 10.23^{* *}$ & $14.53 \pm 0.92^{* *}$ \\
\hline VI & $\begin{array}{c}\text { RNM } \\
\text { (alone) }\end{array}$ & $77.25 \pm 6.75$ & $125 \pm 5.72$ & $47.5 \pm 3.25$ & $58.0 \pm 8.38$ & $11.5 \pm 1.01$ \\
\hline V & $\begin{array}{c}\text { Orilistat } \\
\text { (alone) }\end{array}$ & $69.32 \pm 4.72$ & $98.2 \pm 3.52$ & $40.3 \pm 2.6$ & $42.9 \pm 9.3$ & $10.52 \pm 1.06$ \\
\hline
\end{tabular}

Data represent mean \pm standard deviation (SD). Values are not sharing a common superscript $(*)$ differ significant at $\mathrm{p} \leq 0.05$.

In group- IV the HFD+Orlistat administered, rats did not show any significant alterations in their levels as compared to that of normal animals. GroupV and GroupVI rats were of respective control of RNME and Orlistat, which showed almost equivalent values to normal rats.

The animals of normal and other treatments were weighed for weight gain at the starting of experiment and then to every two weeks till the 6th week of the experimentation. Using the electronic balance, body weight loss or gain of rats that were fed on experimental diets with or without RNM were shown in (Table 2).

Table 2:- Effect of R.nasutus and orlistat on body weight (grams) of rats fed with high fat diets

\begin{tabular}{|c|c|c|c|c|c|c|}
\hline $\begin{array}{c}\text { Obese } \\
\text { markers } \\
\text { (Body weight) }\end{array}$ & control & High fat diet & HFD+RNM & HFD+Orlistat & $\begin{array}{c}\text { RNM } \\
\text { (alone) }\end{array}$ & $\begin{array}{c}\text { Orilistat } \\
\text { (alone) }\end{array}$ \\
\hline $0^{\text {th }}$ week & $158.27 \pm 7.90^{*}$ & $157.52 \pm 8.49^{*}$ & $157.90 \pm 8.64^{*}$ & $158.13 \pm 6.20^{*}$ & $158.1 \pm 7.5$ & $158.0 \pm 7.20$ \\
\hline $2^{\text {nd }}$ week & $188.34 \pm 7.31^{*}$ & $201.79 \pm 6.32^{*}$ & $182.96 \pm 8.32^{*}$ & $179.44 \pm 7.11^{*}$ & $152 \pm 6.85$ & $150 \pm 6.8$ \\
\hline $4^{\text {th }}$ week & $217.10 \pm 8.41^{*}$ & $240.01 \pm 9.71^{*}$ & $212.85 \pm 7.90^{*}$ & $217.80 \pm 11.40^{*}$ & $147 \pm 6.29$ & $145 \pm 6.53$ \\
\hline $6^{\text {th }}$ week & $239.42 \pm 10.0^{*}$ & $280.27 \pm 10.9^{*}$ & $238.60 \pm 8.39^{*}$ & $248.01 \pm 11.74^{*}$ & $140.6 \pm 6.2$ & $135 \pm 6.27$ \\
\hline
\end{tabular}

Data represent mean \pm standard deviation (SD). Values are not sharing a common superscript $(*)$ differ significant at $\mathrm{p} \leq 0.05$ RNM: Rhinacanthus nasutus methonolic extract.

The body weights of rats fed with HFD were significantly increased with time, and percentage increase was $151 \%$ for control, $178 \%$ for high fat fed diet rats, $151 \%$ for HFD and RNM rats, $157 \%$ for Orlistat treated with HFD, $88 \%$ with RNM alone, and $85 \%$ with Orlistat alone were in rats. In contrast, non-significant differences were observed in 
rats. The increase in body weight was 21 to $27 \%$ higher in HFD rats than RNM or Orlistat treated animals which is almost equal to normal, and the loss weight in the presence of RNM and Orlistat alone was ranged from 63 to $66 \%$. All these results are significant with $\mathrm{P} \leq 0.05$.

\section{Discussion:-}

Dietary fat and other risk factors:-

Diet is a source of energy for growth and metabolic activities. Fat is an energy rich molecule used in diet after two macro molecules such as carbohydrate and protein, and fat is essential to maintain the integrity of cell and cell membranes. This molecule under normal conditions is essential and excess intake of it causes several problems to humans. The main ingredients of lipids are free fatty acids, triglycerides, cholesterol, phospholipids and lipoproteins. The lipoproteins may carry cholesterol and also have essential fatty acids. The animals feed containing more of lipids either be consumed during exercise or stored in cells. Because of storage of fatty materials in cells there is possibility to acquire lysosomal lipidosis and due to this storage, the liver and spleen shall be enlarged. These disorders are treated with medications by using compounds like Gabapentin, Carbamazepine, Orlistat, Statins etc. The plant products are also used as medications to treat hyperlipidemia and retain the physical characters.

R. nasutus a well known traditional medicinal plant, possesses diverse biological and pharmacological activities including reducing blood glucose and serum lipids. It has long been used to treat diabetes mellitus and related hyperlipidemia. Hypercholesterolemia, a high cholesterol diet caused oxidative stress which increase serum LDL levels to result in increased risk for development of atherosclerosis.( Chander. et al 2003) [21]. Cholesterol is synthesized in all animal tissue to produce various steroids and aldosterones. Increased amount of cholesterol leads to cardiovascular diseases particularly coronary heart disease (CHD) and coronary artery disease (CAD)( Aparna. et al 2003). [22]. In hyperlipidemia, cholesterol feeding has often been used to elevate serum or tissue cholesterol levels and assess hypercholesterolemia-related metabolic disturbances in different animal models. Ma Angeles. et al 1999) [23]. The humans premature death in India is due to hyperlipidemias and atherosclerosis.

In the present study, feeding of rats with energy rich feed has resulted in increased TC, TG and LDL cholesterol levels (Table.1). This model was used to study the potential of hypolipidemic effect of supplementations of $R$. nasutus that contained significant amounts of antioxidants properties. From this study, we found that the daily oral administration of $R$. nasutus supplement diet showed a positive result on significant reduction of total cholesterol levels in plasma after 6 weeks of administration. This result is in agreement with literature where depleted level of HFD fed hyperlipdemia found in the rats upon treatment of R.nasutus extract.

Low density lipoprotein is a athernogenic risk factor and plays a role in several steps of atherosclerosis. A decrease in oxidative stress and removal of LDL from oxidation might therefore be a strategy with great promise for prevention of atherosclerosis associated cardiovascular disease. The intense interest in this area results in part from the generally low toxicity of antioxidants and the hope that treatment with antioxidants might be additive with cholesterol lowering regimes. The increase of HDL on treatment of R. nasutus may serve HDL as directly antiandrogenic and it is believed to remove cholesterol from the developing lesions.

The HFD treated rats also showed an increase as 2 fold of cholesterol, 1.47 fold of triglycerides, 4.3 fold LDL-C and 1.56 fold of VLDL-C increase and 0.81 fold decrease of HDL-C. However the RNM mixed HFD showed 1.26 fold of cholesterol, 1.22 fold of triglycerides, 1.88 fold of LDL-C, 1.24 fold of VLDL-C decrease, and 1.23 fold increase of HDL-C in serum of blood (Table-1). Hence the mixture of RNM has potential to decrease various fat molecules and increase in HDL moiety.

The small particles less than chylomicrons are VLDL which are rich in triglycerides though to a lesser extent VLDL particles sizes vary widely, with a concomitant variation of the chemical composition. The larger particles are rich in triglycerides and in apo-c, and the smaller particles depletion of TG and surface materials result from the hydrolysis of VLDL by lipoprotein lipase activity. VLDL is the main carrier of triglycerides and it is less harmful than but still can damage the arterial lining. In the present study serum TG levels were significantly elevated in HFD rat. The excess of fat diet increased the TG level which is one of the causes of hardening of arteries. In conclusion, it could be said that the methanolic leaf extract of $R$. nasutus exhibited a significant hypolipidemic activity by increasing VLDL, HDL, and by decreasing TC, TG (free) and LDL in serum of blood. 


\section{Conclusion:-}

Result of present study has revealed that the methanolic extract of $R$. nasutus leaves can improve the serum lipid profile in rats by decreasing serum TC, TG, LDL-C and increasing serum

HDL-C, and thus improving the atherogenic index. These findings provide some biochemical basis for the use of leaf extract of $R$. nasutus as antihyperlipidemic agent because of its preventive and curative effect against hyperlipidemia. Further studies are required to gain more insight into the possible mechanism of action.

\section{References:-}

1. Brai BIC, Odebolt MA, Agoma PU. Effect of Persia Americana leaf extract on body weight and liver lipids in rats fed with hyperlipidemic diet. Afr J Biotechnology 2007; 16(8): 1007-1111.

2. Www.who.int/media centre/fastsheets/fs317/en. Ref.British Heart foundation(2015) Table.1

3. Kearney PM, Whelton M, Reynolds K, Muntner P, Whelton PK, He J. Global burden of hypertension: analysis of worldwide data. Lancet 2005; 365: 217-223.

4. Howard-Alpe GM, Sear JW, Foex P. Methods of detecting atherosclerosis in non-cardiac surgical patients; the role of biochemical markers. Br J Anesthesia 2006; 97: 758-769.

5. Sattivel A, Rao H, Balajiaghavendran. Anti peroxidative and Anti hyperlipidemic nature of Ulva lactuca crude polysaccharide on D-galactose amine induced hepatitis in rats. Food Chem Toxicol 2000; 46: 3262-3267.

6. Durrington P. Dyslipidaemia. Lancet 2003; 362: 717-731.

7. Speight TM. Avery's drug treatment principles and practice of clinical pharmacology and therapeutics. Australia: Maclennan and Petty; 1987.

8. WHO. Resolution-promotion and development of training and research in traditional medicine. Geneva: WHO; 1977, p. 49-49.

9. Harborne JB. Photochemical Methods. A Guide to Modern Techniques of Plant Analysis. 1st Edn., London: Chapman and Hall; 1973.

10. Sofowora A. Medicinal Plant and Traditional Medicine in Africa. 2nd Edn. Ibadan: Spectrum Books; 1996, p. 112.

11. Kumari K, Augusti KT. Lipid lowering effect of S-methyl cysteine sulfoxide from Allium cepa Linn in high cholesterol diet fed rats. J Ethnopharmacol 2007; 109: 367-371.

12. Son IS, Kim JH, Sohn HY, Son KH, Kim JS, Kwon CS. Ant oxidative and hypolipidemic effects of diosgenin, a steroidal saponin of yam (Dioscorea spp.), on high-cholesterol fed rats.

13. Indian medical plants. A compendium of 500 species.Chennai:Orient Longman Ltd:1994;4:417

14. Visweswara rao P, Sivanarayana A,Sasikala S and Dhananjaya M.Efficancy of antimicrobial activity of Rhinachantus Nasutus (Linn) leaves in different exctrations.Int J Pharma Biol Sci.2010 ;(2);1-4

15. Yesilbursad,Serdar Z,SerdarA,SaracM,Coskun S,JaleC. Lipid peroxides in obese patients and effect of weight loss with Orilistat on lipid peroxides levels. International Journal of Obesity and Related Metabolic Disorder2005;29(1)142e5.

16. Srinivasan K, Patole PS, Kaul CL, Ramarao P. Reversal of glucose intolerance by pioglitazone in high-fat diet fed rats. Methods Find Exp Clin Pharmacol 2004; 26: 327-333.

17. Reed MJ, Meszaros K, Entes LJ, Claypool MD, Pinkett JG, Gadbois TM, et al. A new rat model of type 2 diabetes: the fatfed, streptozotocin-treated rat. Metabolism 2000; 49: 1390-1394.

18. Parekh AC, Jung DM. Cholesterol determination with ferric acetate-uranium acetate and sulphuric acid, ferrous sulphate reagents. Anal Chem 1970; 42: 1423-1427.

19. Rice LB. Determination of triglycerides (enzymatic method). Clin Chem 1970; 31(5): 746-750.

20. Lyons TJ. Lipoprotein glycation and its metabolic complications. Diabetes 1992; 41(sup2): 67-73

21. Chander R, Kapoorn $\mathrm{K}$ and Singh C. Lipid per oxidation of hyperlipidemic rat serum in chronic ethanol and acetaldehyde administration. J Biosci 2003; 13: 289-274.

22. Aparna Berteri R. Risk of coronary artery heart disease. Health Screen 2003; 1: 28-29.

23. Ma Angeles Zulet, $\mathrm{PhD}$, Ana Barber, PhD, Henri Garcin, PhD, Paul Higueret, PhD, and Jose' Alfredo Martı'nez, PhD, FACN "Alterations in Carbohydrate and Lipid Metabolism Induced by a Diet Rich in Coconut Oil and Cholesterol in a Rat Model" Journal of the American College of Nutrition, Vol. 18, No. 1, 36-42 (1999). 